\title{
ORIGINAL
}

\section{EVALUACIÓN EN CINCO CIUDADES ESPAÑOLAS DEL IMPACTO EN SALUD DE LA CONTAMINACIÓN ATMOSFÉRICA POR PARTÍCULAS. PROYECTO EUROPEO APHEIS (*)}

\author{
Eva Alonso Fustel (1), Teresa Martínez Rueda (1), Koldo Cambra Contín (1), Laura Lopez \\ Carrasco (2), Elena Boldo Pascua (2), Belén Zorrilla Torras (2), Antonio Daponte Codina (3), \\ Inmaculada Aguilera Jiménez (3), Silvia Toro Cárdenas (3), Carmen Iñiguez Hernandez (4), \\ Ferrán Ballester Diez (4), Francisco García García (4), Antoni Plasencia Taradach (5), Lucía \\ Artazcoz Lazcano (5) y Silvia Medina (6)
}

(1) Departamento de Sanidad del Gobierno Vasco

(2) Instituto de Salud Pública. Madrid

(3) Escuela Andaluza de Salud Pública

(4) Escuela Valenciana de Estudios para la Salud (EVES)

(5) Agència de Salut Pública de Barcelona

(6) Instituto Nacional de Vigilancia de Salud Pública de Francia (InVS)

(*) El proyecto APHEIS está cofinanciado por el Programa de Enfermedades Relacionadas con la Contaminación CG SANCO de la Comisión (contracts n SI2.131174 [99CVF2-604], SI2.297300 [2000CVG2-607] and SI2.326507 [2001CVG2-602]) y los centros participantes. Más información en http://www.apheis.net/.

$\dagger$ Elena Boldo disfrutó de una beca de la Consejería de Sanidad y Consumo, Comunidad de Madrid (Orden 566/2001).

\section{RESUMEN}

Fundamento: Los efectos de la contaminación atmosférica sobre la salud han sido objeto en los últimos años de numerosos estudios que han permitido cuantificar la asociación entre ambas El objetivo de este trabajo es llevar a cabo la Evaluación del Impacto en Salud (EIS) calculando los beneficios que se obtendrían al cumplir los objetivos establecidos por la Directiva 1999/30/CE en relación con las partículas en suspensión.

Métodos: Se ha valorado el impacto en salud de la contaminación atmosférica por partículas en suspensión, para lo que se han utilizado dos indicadores distintos: Humos Negros (HN) (Barcelona, Bilbao, Valencia) y partículas en suspensión menores de $10 \mu \mathrm{m}$ $\left(\mathrm{PM}_{10}\right)$ (Bilbao, Madrid y Sevilla). Los indicadores de salud fueron la mortalidad por todas las causas, por causa respiratoria y por causa cardiovascular, e ingresos hospitalarios urgentes por causa respiratoria y cardiovascular. El EIS se ha realizado mediante el cálculo de la fracción atribuible a la contaminación por partículas. En el caso de PM10 se ha calculado el impacto debido a efectos de la contaminación a corto plazo, los acumulados hasta 40 días después, y a largo plazo. Para HN únicamente se han calculado efectos a corto plazo.

Correspondencia:

Eva Alonso Fustel

Unidad de Vigilancia Epidemiológica

Subdirección de Salud Pública de Bizkaia

C/ Ma Díaz de Haro n ${ }^{\circ} 6048010$ Bilbao

Correo electrónico: tepidebi-san@ej-gv.es

\section{ABSTRACT}

\section{Health Impact Evaluation of Particle Air Pollution in Five Spanish Cities. European APHEIS Project}

Background: The health effects of air pollution have been analyzed in numerous studies over recent years, thus having made it possible to quantify the relationship between the two. This study is aimed at analyzing the theoretical benefits which would be achieved by meeting the air quality objectives set forth under EC Directive 1999/30/EC with regard to suspended particles.

Methods: The exposure measurement was taken for Black Smoke (Barcelona, Bilbao, Valencia) and suspended particles under 10 $\mu \mathrm{m}\left(\mathrm{PM}_{10}\right)$ (Bilbao, Madrid y Sevilla). The health indicators calculated were the mortality due to all causes and respiratory and cardiovascular causes, and emergency hospital admissions and mortality due to respiratory and cardiovascular causes. In the case of $\mathrm{PM}_{10}$ the impact has been calculated because its effects to short-term, within a period of up to 40 days following exposure, and to long-term. For Black Smoke the effects only has been calculated to short-term.

Results: The daily levels of $\mathrm{PM}_{10}$ from exceeding $50 \mu \mathrm{g} / \mathrm{m}^{3}$ in Bilbao, Madrid and Sevilla cause the earlier death of $1.4 / 100,000$ individuals per year because its effects. The effect within a period of up to 40 days following exposure is of 2.8 deaths $/ 100,000$. The total number of deaths per year which may be later due to long-term exposure if the yearly average is lowered to $20 \mu \mathrm{g} / \mathrm{m}^{3}$ is $68 / 100,000$. 
Resultados: Los niveles diarios de $\mathrm{PM}_{10}$ por encima de 50 $\mu \mathrm{g} / \mathrm{m}^{3}$ en Bilbao, Madrid y Sevilla son responsables de 1,4 muertes prematuras por 100.000 habitantes y año debido a sus efectos a corto plazo y de 2,8 muertes/100.000 en un periodo de hasta 40 días tras la exposición. A largo plazo, el número de muertes prematuras atribuibles a la contaminación media anual de $\mathrm{PM}_{10}$ por encima de 20 $\mu \mathrm{g} / \mathrm{m}^{3}$ es $68 / 100.000$.

Conclusiones: El impacto en salud de los niveles actuales de contaminación atmosférica es cuantificable y no despreciable. APHEIS y las evaluaciones de impacto pueden ayudar a la planificación sanitaria y a las políticas medioambientales.

Palabras clave: Contaminación atmosférica. Medio ambiente y salud pública. Causa de muerte.

\section{INTRODUCCIÓN}

Los efectos de la contaminación atmosférica sobre la salud han sido objeto en los últimos años de numerosos estudios que han permitido cuantificar la asociación entre ambas $^{1,2}$. Aplicando técnicas analíticas que permiten relacionar las defunciones o los ingresos hospitalarios con las concentraciones de los contaminantes se han obtenido asociaciones estadísticamente significativas entre la morbimortalidad y las partículas presentes en el aire en estudios a corto (series temporales) y a largo plazo (cohortes). Los diseños de cohorte longitudinales ${ }^{3}$ permiten estimar los efectos crónicos de la exposición ajustando los riesgos por factores individuales, aunque siguen dependiendo de medidas agregadas de exposición (estudios ecológicos). Los estudios de series temporales estiman la probabilidad de morir prematuramente o de enfermar debido a la exposición reciente a la contaminación atmosféri$\mathrm{ca}^{4,5}$. En España, en el año 1997 se realizó el estudio de series temporales Estudio Multicéntrico Español de Contaminación Atmosférica y Mortalidad (EMECAM) $)^{6-8} \mathrm{y}$ en el año 2000 Estudio Multicéntrico Español de Contaminación Atmosférica y Salud (EMECAS $)^{9,10}$.

Muchos estudios, entre ellos los dos españoles citados anteriormente, han mostrado de manera clara que existe asociación entre los niveles de contaminantes atmosféricos (especialmente las partículas en suspensión) $\mathrm{y}$ una serie de indicadores de salud, como la
Conclusions: The health impact of the current air pollution levels is quantifiable and is not insignificant. APHEIS and the impact evaluations may be of aid in healthcare planning and environmental policies.

Key words: Air pollution. Environment and Public Health. Cause of death. mortalidad o los ingresos hospitalarios. La evaluación del impacto en salud (EIS) de un contaminante tiene sentido cuando existe una evidencia suficiente de que su relación con el efecto medido es de tipo causal. En el caso de las partículas en suspensión la naturaleza causal de la asociación viene avalada por la consistencia de los resultados obtenidos en distintos periodos y lugares con un amplio rango de condiciones ambientales, por diferentes investigadores y utilizando distintos métodos analíticos. Además, los estudios toxicológicos experimentales han proporcionado información sobre los mecanismos biológicos por los que actúan estos contaminantes ${ }^{11}$.

Sin embargo, a pesar de la consistencia de las asociaciones entre partículas en suspensión y varios indicadores de efectos en salud, su significado o trascendencia para la salud pública no es siempre fácil de interpretar. En el año 1999 se realizó el programa APHEIS (Contaminación del aire y salud. Un sistema europeo de información) con la finalidad de proporcionar información accesible, completa y actualizada sobre el impacto de la contaminación atmosférica en la salud pública a los responsables políticos, profesionales de la salud ambiental y a los ciudadanos europeos. Se trata de un proyecto con 26 centros participantes de 11 países que se ha llevado a cabo durante el período 20002003. Uno de sus objetivos fundamentales es valorar el impacto en la salud mediante el cálculo del número de ingresos hospitalarios, del número de muertes y de la reduc- 
ción de la esperanza de vida atribuibles a la contaminación atmosférica. La finalidad de este artículo es dar a conocer la metodología del proyecto y presentar los resultados en las 5 ciudades españolas participantes: Barcelona, Bilbao, Madrid, Sevilla y Valencia.

\section{MATERIAL Y MÉTODOS}

Las cinco ciudades españolas participantes en APHEIS suman una población total de 6.603.617 habitantes. La valoración de los efectos de la contaminación de los humos negros se ha realizado para 2.964.179 personas y la de partículas $\mathrm{PM}_{10}$, para 4.347.832. (tabla 1).

APHEIS desarrolló 5 guías metodológicas orientadas a la EIS de la contaminación del aire (Evaluación de la exposición, Salud Pública, Epidemiología, Cálculo de Impacto y Estadística $)^{13}$ a partir de las recomendaciones de la $\mathrm{OMS}^{14,15}$.

Medida de la Exposición: Las Redes de Vigilancia de la calidad del aire establecidas en las ciudades miden diferentes fracciones de la contaminación atmosférica por partículas: partículas de diámetro inferior a $10 \mu \mathrm{m}$ $\left(\mathrm{PM}_{10}\right)$ o $2,5 \mu \mathrm{m}\left(\mathrm{PM}_{2,5}\right)$ y humos negros $\mathrm{HN}$. Se han utilizado los datos de 47 captadores urbanos que cumplían los requisitos establecidos en la guía de Medición de la Exposición de APHEIS ${ }^{13}$. Barcelona, Bilbao y Valencia han dispuesto de datos de Humos Negros (HN) y Bilbao, Madrid y Sevilla datos de partículas menores de $10 \mu \mathrm{m}\left(\mathrm{PM}_{10}\right)$. (tabla 1). Ninguna de las ciudades contaba con suficientes mediciones de $\mathrm{PM}_{2.5}$. Los datos de $\mathrm{PM}_{10}$ de los captadores automáticos fueron corregidos mediante factores para compensar las pérdidas por volatilidad de las partículas ${ }^{16}$. A partir de los datos de $\mathrm{PM}_{10}$ se calculó la fracción de partículas de diámetro menor que 2,5 $\mu \mathrm{m}\left(\mathrm{PM}_{2, .5}\right)$, necesaria para poder valorar algunos efectos en salud para los que únicamente existen RR disponibles para las partículas de ese tamaño. De mane- ra general se ha estimado que las $\mathrm{PM}_{2.5}$ representan el $70 \%$ de las $\mathrm{PM}_{10}{ }^{17}$. En el caso de Madrid se utilizó un factor de 0,5 pues datos de mediciones locales indicaban que la proporción de $\mathrm{PM}_{2.5}$ respecto a las de $\mathrm{PM}_{10}$ era del $50 \%$.

Indicadores de salud: Se han valorado los efectos a corto plazo (1 día y acumulados hasta 40 días) sobre la mortalidad por todas las causas salvo las externas (ICD10: A00R99), por causas cardiovasculares (ICD 10:I00-I99) y respiratorias (ICD10:J00-J99), así como los ingresos hospitalarios urgentes por causas cardiacas (ICD 10: I00-I52) y respiratorias (ICD10:J00-J99). Para los efectos a largo plazo, se han empleado la mortalidad por todas las causas (ICD10:A00-Y98), por causas cardiopulmonares (ICD10:I10-I70 y J00-J99) y cáncer de pulmón (ICD10:C33C34). Los valores diarios de mortalidad se han extraído de los registros de mortalidad de las Comunidades Autónomas a las que pertenecen las ciudades participantes y los ingresos hospitalarios urgentes proceden de los registros de altas hospitalarias.

\section{Cálculo del impacto}

El impacto en salud se ha basado en el cálculo de las fracciones de la mortalidad y de los ingresos hospitalarios atribuibles a la contaminación atmosférica por partículas. Para ello es necesario especificar un nivel mínimo de exposición a la contaminación (escenario de referencia), pues la fracción atribuible se calcula para el riesgo derivado de la exposición a los contaminantes por encima de ese valor mínimo. APHEIS ha tomado como escenario de referencia los planteados como objetivo de calidad de $\mathrm{PM}_{10}$ para 2010 en la Directiva 1999/30/CE, y que son:(1) la eliminación de los niveles diarios de $\mathrm{PM}_{10}$ por encima de $50 \mu \mathrm{g} / \mathrm{m}^{3}$; (2) la reducción de la media anual de $\mathrm{PM}_{10}$ a 20 $\mu \mathrm{g} / \mathrm{m}^{3}$; y (3) la reducción de $5 \mu \mathrm{g} / \mathrm{m}^{3}$ en los niveles diarios que presente cada ciudad. 


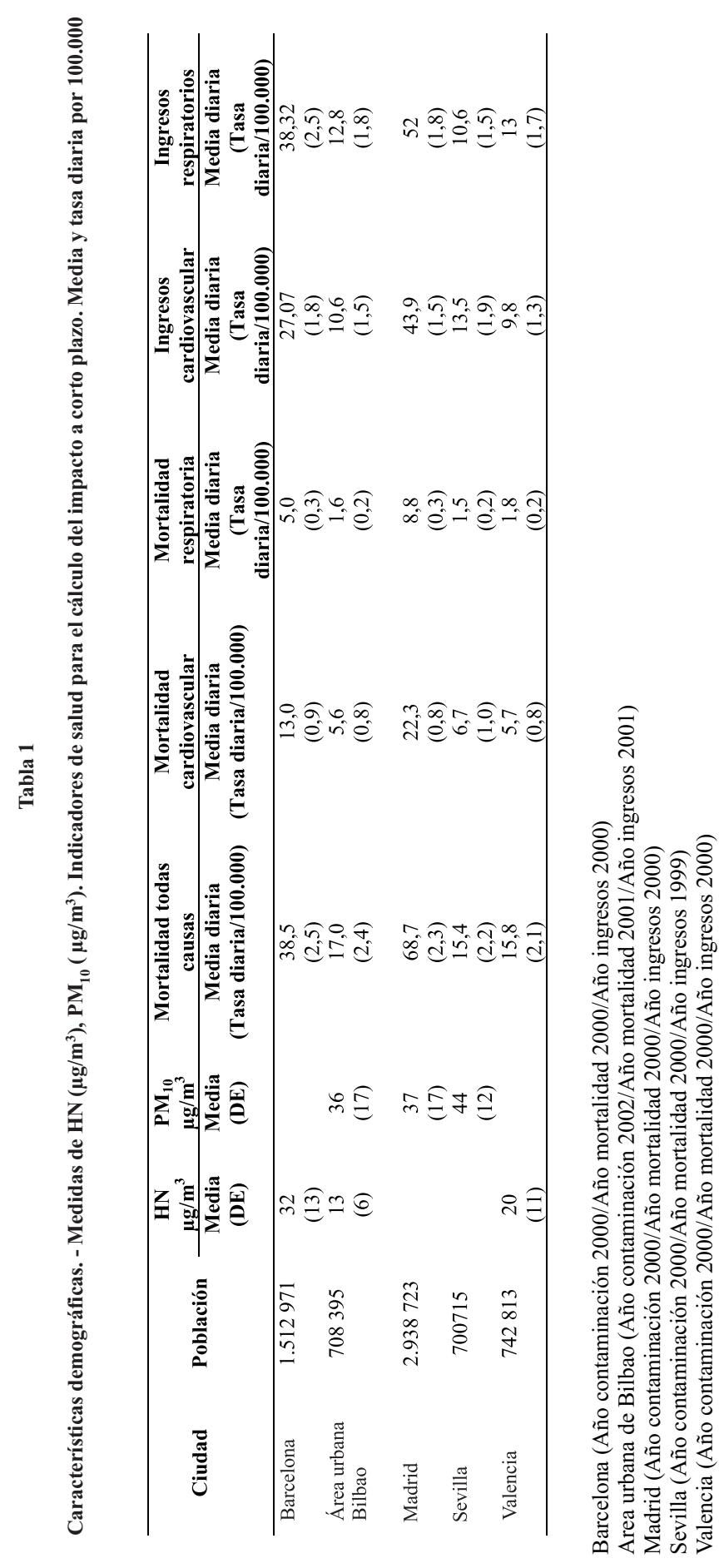


Los impactos calculados en relación con los escenarios 1 y 2 representan el beneficio que se obtendría si se cumplen los objetivos de calidad marcados en la norma.

Aunque los niveles de $\mathrm{HN}$ no se recogen en la citada directiva la evaluación del impacto se ha realizado por similitud para dos escenarios: (1) la eliminación de los niveles diarios por encima de $20 \mu \mathrm{g} / \mathrm{m}^{3}$ y (2) reducción diaria de $5 \mu \mathrm{g} / \mathrm{m}^{3}$.

Para $\mathrm{HN}$ únicamente se han calculado efectos a corto plazo. En el caso de $\mathrm{PM}_{10}$ se ha calculado el impacto debido a efectos de la contaminación a corto plazo (en el día siguiente a la exposición), los acumulados hasta 40 días después, y a largo plazo. La evaluación de cada uno de ellos requiere lógicamente el uso de un RR específico. La mayor parte de los RRs utilizados para valorar los efectos al día siguiente de la exposición a $\mathrm{HN}$ y $\mathrm{PM}_{10}$ proceden de los resultados del estudio multicéntrico europeo APHEA $^{22}$. APHEIS llevó a cabo un metaanálisis y calculó una función exposición respuesta para los ingresos hospitalarios por causas respiratorias ${ }^{18}$. Los RRs para los efectos acumulados hasta 40 días proceden de los estudios de Zanobetti ${ }^{19,20}$ mientras que para los efectos a largo plazo se han empleado los resultados de los estudios de cohortes norteamericanos donde las partículas vienen expresadas como $\mathrm{PM}_{25}$ (tabla 2). Se ha asumido que la relación dosis-respuesta es lineal, lo que equivale a que una variación lineal dada en la contaminación por partículas en suspensión (incremento o disminución) tiene siempre las mismas consecuencias para la salud de la población, con independencia del nivel de contaminación al que ocurra ${ }^{21,22}$.

En el cálculo del número de muertes atribuibles (MA) (para los ingresos es exactamente lo mismo) se ha asumido que toda la población está expuesta a la contaminación atmosférica, y que el indicador calculado es un buen estimador de la exposición media de sus habitantes, por lo que la fórmula de cál- culo se simplifica a $\mathrm{MA}=\left[\left(\mathrm{RR}_{\Delta}-1\right) / \mathrm{RR}_{\Delta}\right] \mathrm{M}$; donde $\mathrm{M}=$ número total de muertes; $\mathrm{RR}_{\Delta}$ $=\mathrm{RR}$ para el diferencial de contaminación entre la situación actual y el nivel del escenario de referencia. Los cálculos se han realizado con una hoja de cálculo en Excel llamada PSAS- $9{ }^{23}$ y diseñada por el Sistema de Vigilancia en Contaminación Atmosférica y Salud en Francia. En el caso de los efectos a largo plazo se ha calculado además el impacto de la contaminación atmosférica en la esperanza de vida a la edad de 30 años, que estima en qué medida son adelantadas las muertes atribuibles. Para ello se ha empleado el programa Air Q de la OMS (WHOECEH Air Quality Health Impact Assessment software $)^{24}$. El método seguido se basa en el cálculo de las funciones de supervivencia de la población con el riesgo de la contaminación atmosférica y sin él, a partir de los RR de efectos a largo plazo de la contaminación y las distribuciones de población y mortalidad por edades de cada población

\section{RESULTADOS}

La media diaria de los niveles de contaminación por humos negros oscila entre 13 $\mu \mathrm{g} / \mathrm{m}^{3}$ en Bilbao y $32 \mu \mathrm{g} / \mathrm{m}^{3}$ en Barcelona. Los valores de $\mathrm{PM}_{10}$ son casi iguales en Bilbao y Madrid y algo más elevados en Sevilla. Estas tres ciudades superan el valor límite anual establecido en la Directiva 1999/30/EC para el año $2010\left(20 \mu \mathrm{g} / \mathrm{m}^{3}\right)$ (tabla 1).

En las ciudades de Barcelona, Bilbao y Valencia el número de muertes atribuible a los efectos a corto plazo de los niveles diarios de $\mathrm{HN}$ por encima de $20 \mu \mathrm{g} / \mathrm{m}^{3}$ son 101 $(3,4 / 100.000)$. Los ingresos atribuibles a estos mismos niveles de contaminación son 123 por causa cardiaca y 47 por enfermedad respiratoria (tabla 3 ).

Del mismo modo en Bilbao, Madrid y Sevilla los niveles diarios de $\mathrm{PM}_{10}$ por encima de $50 \mu \mathrm{g} / \mathrm{m}^{3}$ son responsables de 59 
Tabla 2

Indicadores de salud, RR (IC95\%)

\begin{tabular}{|c|c|c|c|}
\hline & $\begin{array}{l}\text { Indicador de salud } \\
\text { Efectos a corto plazo }\end{array}$ & $\begin{array}{l}\text { RR (IC95\%) para } \uparrow \\
\text { de } 10 \mu \mathrm{g} / \mathrm{m} 3\end{array}$ & Ref. Bibliográfica \\
\hline \multirow{5}{*}{$\begin{array}{l}\text { Humos } \\
\text { negros }\end{array}$} & Mortalidad por todas causas excluyendo externas & $1,006(1,004-1,009)$ & WHO $2004^{2}$ \\
\hline & Mortalidad cardiovascular & $1,004(1,002-1,007)$ & WHO $2004^{2}$ \\
\hline & Mortalidad respiratoria & $1,006(0,998-1,015)$ & WHO $2004^{2}$ \\
\hline & Ingresos hospitalarios urgentes cardiacas & $1,011(1,004-1,019)$ & Le Tertre et al. $2002^{4}$ \\
\hline & Ingresos hospitalarios urgentes respiratorias & $1,003(0,999-1,008)$ & APHEIS $3^{18}$ \\
\hline \multirow[t]{5}{*}{$\mathrm{PM}_{10}$} & Mortalidad por todas causas excluyendo externas & $1,006(1,004-1,008)$ & WHO $2004^{2}$ \\
\hline & Mortalidad cardiovascular & $1,009(1,005-1,013)$ & WHO $2004^{2}$ \\
\hline & Mortalidad respiratoria & $1,013(1,005-1,021)$ & WHO $2004^{2}$ \\
\hline & Ingresos hospitalarios urgentes cardiacas & $1,006(1,003-1,009)$ & Le Tertre et al. $2002^{4}$ \\
\hline & Ingresos hospitalarios urgentes respiratorias & $1,011(1,006-1,017)$ & APHEIS $3^{18}$ \\
\hline \multirow[t]{3}{*}{$\mathrm{PM}_{\mathrm{t} 0} 40$ días } & Mortalidad por todas causas excluyendo externas & $1,012(1,008-1,016)$ & Zanobetti et al,2002 ${ }^{19}$ \\
\hline & Mortalidad cardiovascular & $1,020(1,014-1,026)$ & Zanobetti et al, $2003^{20}$ \\
\hline & Mortalidad respiratoria & $1,042(1,011-1,074)$ & Zanobetti et al, $2003^{20}$ \\
\hline $\mathrm{PM}$ & Efectos a largo plazo & & \\
\hline \multirow[t]{4}{*}{$\mathrm{PM}_{2, s}$} & Mortalidad por todas causas excluyendo externas & $1,043(1,026-1,11)$ & Kunzli et al, $2000^{29}$ \\
\hline & Mortalidad por todas causas & $1,06(1,02-1,11)$ & Pope, $2002^{3}$ \\
\hline & Mortalidad cardiopulmonar & $1,09(1,03-1,16)$ & \\
\hline & Mortalidad por cáncer de pulmón & $1,14(1,04-1,23)$ & \\
\hline
\end{tabular}

Tabla 3

Beneficios potenciales de la reducción diaria de $5 \mu \mathrm{g} / \mathrm{m}^{3}$ de los niveles de $\mathrm{HN}$ y de la reducción de los días que superan $20 \mu \mathrm{g} / \mathrm{m}^{3}$ a $20 \mu \mathrm{g} / \mathrm{m}^{3}$. Número absoluto y número por 100.000 habitantes e IC $95 \%$. Barcelona, Bilbao y Valencia.

\begin{tabular}{|c|c|c|}
\hline \multirow[b]{2}{*}{ Humos negros } & \multicolumn{2}{|c|}{ Mortalidad por todas las causas } \\
\hline & $\mathrm{N}^{0}$ casos atribuibles & $\mathrm{N}^{0}$ casos atribuibles $/ 100.000$ \\
\hline $5 \mu \mathrm{g} / \mathrm{m}^{3}$ & $69,2(46,1-103,6)$ & $2,3(1,6-3,5)$ \\
\hline \multirow[t]{3}{*}{$<=20 \mu \mathrm{g} / \mathrm{m}^{3}$} & $101,2(67,3-152,2)$ & $3,4(2,3-5,1)$ \\
\hline & \multicolumn{2}{|c|}{ Número atribuible de ingresos hospitalarios urgentes } \\
\hline & Enfermedad cardiaca & Enfermedad respiratoria \\
\hline $5 \mu \mathrm{g} / \mathrm{m}^{3}$ & $81,3(33,4-136,5)$ & $30,1(-7-75,4)$ \\
\hline$<=20 \mu \mathrm{g} / \mathrm{m}^{3}$ & $123,3(53,9-204,7)$ & $46,8(-23,2-117,6)$ \\
\hline
\end{tabular}

muertes al año (1,4/100.000 personas) en las 24 horas siguientes a la exposición a la contaminación; el 50,7\% (0,7/100.000) por causas cardiovasculares y el $26 \%(0,4 / 100.000)$ por causas respiratorias. Asimismo serían origen de 83,2 ingresos hospitalarios urgentes por causa respiratoria y 39,2 por causa cardiaca (tabla 4). El efecto de la contaminación en la mortalidad en los 40 días posteriores a la exposición es prácticamente el doble en la mortalidad total (2,8 muertes/100.000) y el triple en el caso de las causas respiratorias $(1,2 / 100.000)$.
Los efectos a largo plazo sólo pueden ser calculados para las ciudades que cuentan con datos de partículas $\mathrm{PM}_{10}$. El número total de muertes por año que son atribuibles a la contaminación media anual por encima de $20 \mu \mathrm{g} / \mathrm{m}^{3}$ es de 68 por 100.000 , lo que supone en las tres ciudades 2.956 muertes por año (tabla 5). De ellas 26,4/100.000 serían por causa cardiopulmonar y 4,6/100.000 por cáncer de pulmón (tabla 5), de manera aproximada puesto que $15 \mu \mathrm{g} / \mathrm{m}^{3}$ de $\mathrm{PM}_{25}$ no equivale exactamente a $20 \mu \mathrm{g} / \mathrm{m}^{3}$ de $\mathrm{PM}_{10}$. La reducción en la esperanza de vida a 


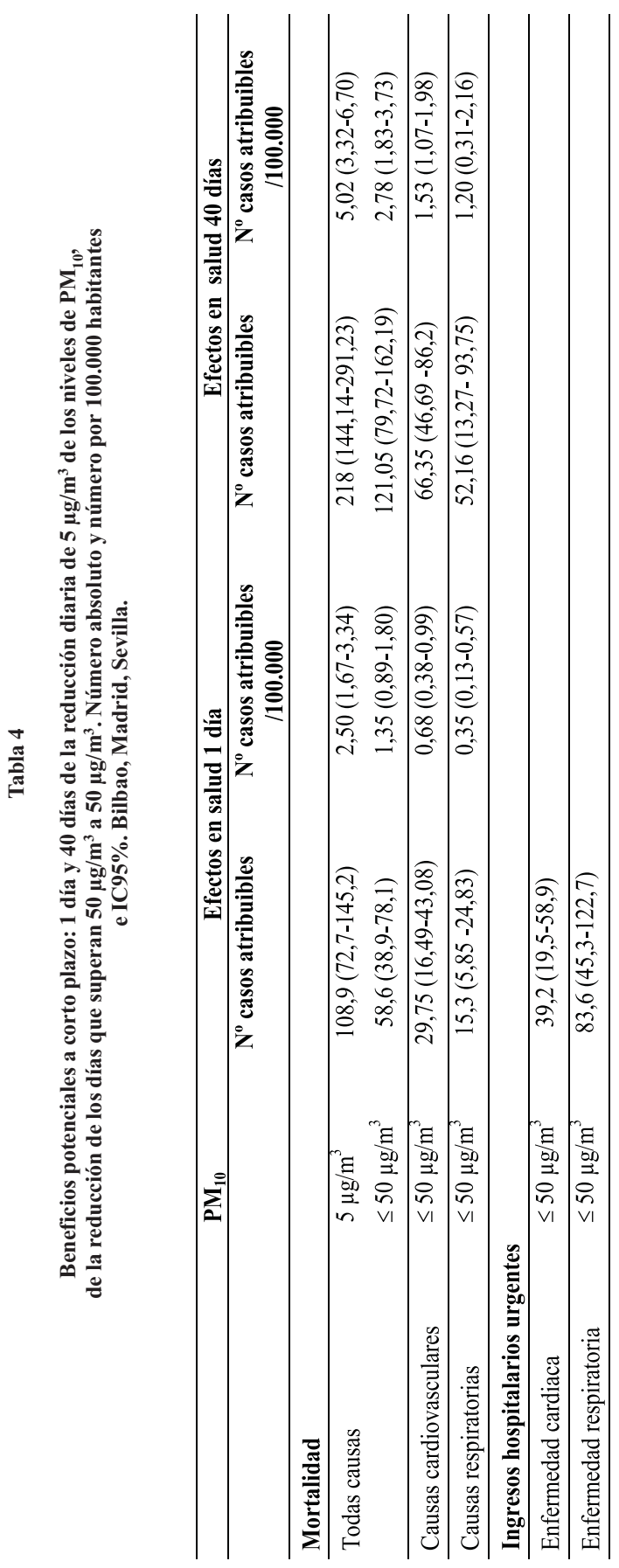


Tabla 5

Beneficios potenciales a largo plazo de la reducción de los valores medios anuales de los niveles de $\mathbf{P M}_{10} \mathbf{y}_{\mathbf{P M}} \mathbf{M}_{2,}$ Número absoluto y número por 100.000 habitantes e IC95\%. Bilbao, Madrid, Sevilla

\begin{tabular}{llcc}
\hline & & \multicolumn{2}{c}{ Efectos en salud a largo plazo } \\
& $\mathbf{P M}_{\mathbf{1 0}}$ & $\mathbf{N}^{\mathbf{0}}$ casos atribuibles & $\mathbf{N}^{\mathbf{0}}$ casos atribuibles/100.000 \\
\hline Mortalidad por todas causas & $5 \mu \mathrm{g} / \mathrm{m}^{3}$ & $772,9(469,3-1091,8)$ & $17,78(10,79-25,11)$ \\
& $\leq 20 \mu \mathrm{g} / \mathrm{m}^{3}$ & $2956,2(1771,1-4234,5)$ & $67,99(40,74-97,39)$ \\
\hline & $\mathbf{P M}_{\mathbf{2 , 5}}$ & & \\
\hline Mortalidad cardiopulmonar & $3,5 \mu \mathrm{g} / \mathrm{m}^{3}$ & $504,18(180,96-834,71)$ & $11,60(4,16-19,20)$ \\
& $\leq 15 \mu \mathrm{g} / \mathrm{m}^{3}$ & $1149,73(400,61-1962,89)$ & $26,44(9,21-45,15)$ \\
\hline Mortalidad por cáncer de pulmón & $3,5 \mu \mathrm{g} / \mathrm{m}^{3}$ & $92,84(31,23-156,34)$ & $2,14(0,72-3,60)$ \\
& $\leq 15 \mu \mathrm{g} / \mathrm{m}^{3}$ & $200,51(64,73-352,58)$ & $4,61(1,49-8,11)$ \\
\hline
\end{tabular}

Figura 1

Número de muertes por todas las causas por 100.000 habitantes, prevenibles al reducir $5 \mu \mathrm{g} / \mathrm{m}^{3}$ en los niveles de $\mathrm{PM}_{10}$ en Bilbao, Madrid, Sevilla a corto plazo, acumulado en 40 días y a largo plazo

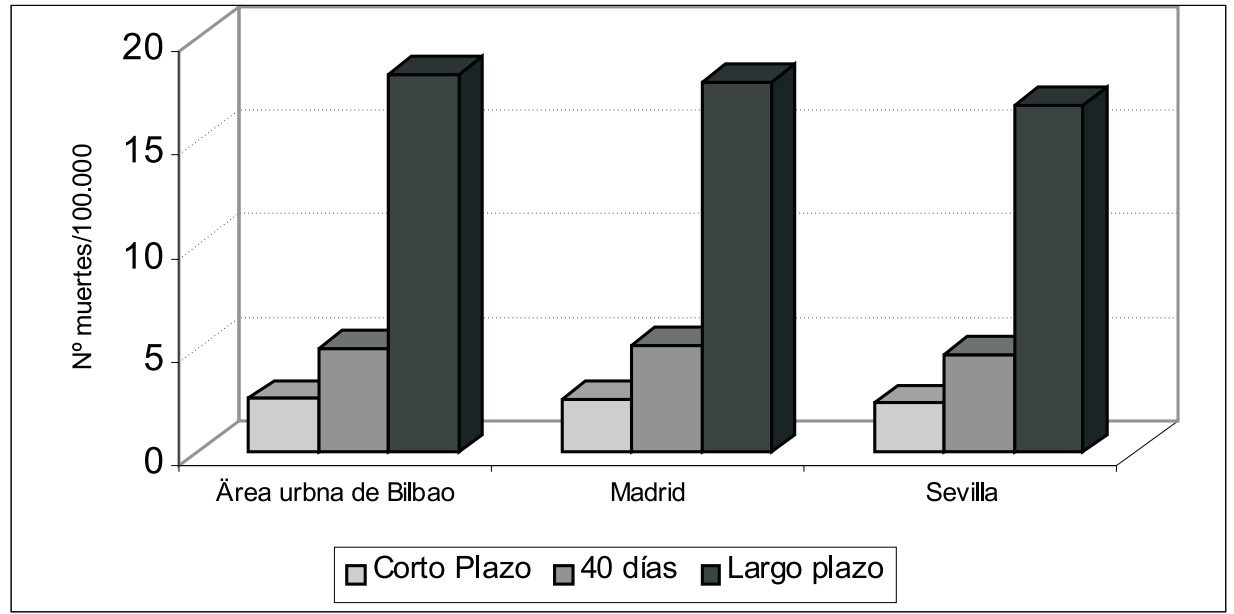

la edad de 30 años atribuible a los niveles de $\mathrm{PM}_{2.5}$ superiores a $15 \mu \mathrm{g} / \mathrm{m}^{3}$ es de 0,22 años $(0,06-0,38)$ en Madrid; $0,90(0,24-1,56)$ en Bilbao y $1,17(0,31-2,04)$ en Sevilla.

En la figura 1 se ilustra el beneficio que se obtendría al reducir la contaminación diaria en $5 \mu \mathrm{g} / \mathrm{m}^{3}$ (lo que implicaría una reducción de la media anual similar) en relación con los efectos en un día, en 40 días y a largo plazo.

\section{DISCUSIÓN}

Las medias diarias de mortalidad por 100.000 habitantes son muy similares en todas las ciudades. La media diaria de los ingresos por causas respiratorias es superior a la media de los de causas cardiacas en todas las ciudades excepto en Sevilla.

Los datos de contaminación empleados han procedido de estaciones de medida 
seleccionadas que representan de manera aceptable la exposición de la población a los contaminantes de aire, y los niveles hallados han sido similares a las ciudades europeas de su entorno ${ }^{26}$. Sin embargo, han existido dos limitaciones reseñables en relación con la especificación de la exposición: el uso de indicadores de partículas distintos $(\mathrm{HN}$ y $\mathrm{PM}_{10}$ ), que hace que los resultados no sean comparables en las 5 ciudades, y la falta de datos medidos de $\mathrm{PM}_{2,5}$. Aunque en la actualidad los estudios que evalúan los efectos en salud de las partículas emplean en su mayoría las partículas medidas como $\mathrm{PM}_{10} \mathrm{y}$ $\mathrm{PM}_{2.5}$, los estudios que han empleado $\mathrm{HN}$ han proporcionado información válida, aunque quizá menos apropiada para la derivación de funciones dosis-respuesta, debido a la incertidumbre en la relevancia en términos de salud de las partículas tal como se miden por el método de $\mathrm{HN}$, lo que se ve reflejado en el hecho de que algunos de los RRs estimados y empleados en la EIS no sean estadísticamente significativos.

Por otra parte, en un estudio de cohortes realizado en Holanda se ha encontrado asociación de magnitud importante entre la exposición a la contaminación atmosférica procedente del tráfico y efectos en la mortalidad por causa cardiovascular ${ }^{27}$. En las ciudades españolas una fuente importante de contaminación por partículas es el tráfico ${ }^{28}$ lo que hace que una parte sustantiva de los efectos causados por la contaminación atmosférica pueda ser atribuida al tráfico motorizado ${ }^{29}$.

Aunque el índice de humos negros se sigue considerando un buen indicador del tráfico, en nuestro estudio únicamente se han evaluado los efectos de HN a corto plazo, pues no existen estudios que hayan determinado RRs para sus efectos crónicos o a largo plazo. A diferencia de las $\mathrm{PM}_{10}$, cuyos escenarios de evaluación se han basado en los objetivos marcado por la Directiva 1999/30/ $\mathrm{EC}^{12}$, no existe una norma que haya establecido objetivos de calidad a medio o largo plazo, y sus escenarios de valoración se han determinado por paralelismo con los de $\mathrm{PM}_{10}$. Los estimadores obtenidos para la mortalidad diaria parecen menores que los provocados por las PM10. El RR de HN para los ingresos hospitalarios urgentes por causa cardiovascular es mayor que el RR por causa respiratoria, lo que se traduce en un mayor número de ingresos atribuibles por la primera causa, a diferencia de lo que ocurre con $\mathrm{PM}_{10}$.

El número calculado de muertes atribuibles a las $\mathrm{PM}_{10}$ aumenta a medida que aumenta la ventana temporal de observación. El número total de muertes atribuible a los efectos de las partículas en las 24 horas siguientes a la exposición es del orden de la mitad que los producidos en los 40 días siguientes, y en torno al $15 \%$ de los efectos a largo plazo. La reducción en la esperanza de vida atribuible a la contaminación es un indicador fácilmente interpretable que integra el número de muertes atribuibles y la medida en que las muertes han sido adelantadas. Los resultados obtenidos en APHEIS se caracterizan por ser de magnitud importante y variar en las tres ciudades. Son los primeros resultados obtenidos en nuestro entorno y existen varias razones para ser prudente en su utilización. La magnitud de la reducción en la esperanza de vida depende del nivel de contaminación en cada ciudad, del RR empleado y de la distribución por edades de la mortalidad. Por el momento los RR para efectos de las partículas en la mortalidad a largo plazo proceden de estudios de cohortes norteamericanos, pues en Europa no se han realizado estudios comparables.

La variabilidad de los resultados de reducción de la esperanza de vida entre ciudades está reflejando incertidumbres en el proceso de valoración. La razón principal que puede explicar las diferencias entre los resultados de Bilbao y Sevilla que no contaban con factores de conversión procedentes de estudios locales, con los de Madrid es el uso de factores distintos para la conversión de $\mathrm{PM}_{10}$ en 
$\mathrm{PM}_{2,5}$. La posibilidad de utilizar en un futuro próximo mediciones reales de $\mathrm{PM}_{2,5}$ despejará esta incertidumbre.

El impacto en salud de los niveles de partículas existentes es cuantificable y no despreciable. La evaluación del impacto en salud puede ser una importante aportación a la valoración de las políticas medioambientales. El número de muertes prematuras atribuibles a la contaminación media anual de $\mathrm{PM}_{10}$ por encima de $20 \mu \mathrm{g} / \mathrm{m}^{3}$ representa una medida del beneficio en salud que tendrá el cumplimiento del objetivo de calidad de la Directiva 1999/30/CE para 2010. Son todavía necesarias investigaciones futuras para evaluar y comparar el impacto de distintos riesgos ambientales.

APHEIS es un ejemplo en el que los resultados de la investigación epidemiológica de un factor de riesgo ambiental se pueden aplicar al cálculo de su impacto en la salud de la población de un área determinada, creando una información útil para la vigilancia de la salud pública, la planificación sanitaria y la formulación de políticas ambientales tanto a nivel local, regional y europeo.

\section{Componentes de los grupos APHEIS por ciudades}

Bilbao: Koldo Cambra (coordinador), Teresa Martínez, Francisco Cirarda, Eva Alonso, Luis González de Galdeano (Departamento de Sanidad del Gobierno Vasco), Mariví Albizu. Alberto Jiménez de Aberasturi (Departamento de Ordenación del Territorio y Medio Ambiente del Gobierno Vasco).

Madrid: Mercedes Martínez (Coordinadora), Servicio de Análisis e Intervención. Dirección General de Salud Pública Alimentación y Consumo. Ana Gandarillas, Servicio de Epidemiología. Instituto de Salud Pública. Dirección General de Salud Pública Alimentación y Consumo. Laura Crespo,
Dirección General de Calidad y Evaluación ambiental. Consejería de Medio Ambiente

Sevilla. Antonio Daponte, Inmaculada Aguilera, Silvia Toro, Ricardo Ocaña.

Valencia: Ferrán Ballester (coordinador), Carmen Iñiguez, Francisco García, Santiago Pérez-Hoyos, Carmela Moya García, Paz Rodríguez. (Escuela Valenciana de Estudios para la Salud. EVES), José Luis Bosch Reig, José Luis Pisón Garcés, José Márquez Pérez, Caridad Sánchez Gracia, Victoria Gallego Rodriguez, (Concejalía de Sanidad y Consumo del Ayuntamiento de Valencia).

Barcelona: Antoni Plasència., Lucia Artazcoz, Joan Güix, Pau Rodríguez, Montserrat Solé, Eduard Mata, Elvira Torné, Joseph Gràcia, Carmen Borrell, Jordi Sunyer, Jordi Vila, Josep María Antó, Marc Sáez.

\section{BIBLIOGRAFÍA}

1. Katsouyanni K, Touloumi G, Samoli E, Gryparis A, Le Tertre A, Monopolis Y et al. Confounding and effect modification in the short term effects of ambient particles on total mortality: Results from 29 European cities within the APHEA 2 project. Epidemiology 2001; 12:521-31.

2. Anderson R, Atkinson R, Peacock JL, Marston L and Konstantinou K Metaanalysis of time-series and panel studies on Particulate Matter and Ozone (O3). WHO Task Group. Copenhagen: WHO Regional Office for Europe;2004 (EUR/04/5042688).

3. Pope A, Burnett R, Thun M, Calle E, Krewski G, Ito $\mathrm{K}$ et al. Lung cancer, cardiopulmonary mortality and long-term exposure to fine particulate air pollution. JAMA 2002; 287:1132-41.

4. Le Tetre A, Medina S, Samoli E, Forsberg B, Michelozzi P, Boumghar A et al. Short-term effects of particulate air pollution on cardiovascular diseases in eight European cities. J Epidemiol Community Health 2002; 56: 773-9.

5. Atkinson, R, Anderson R, Sunyer J, Ayres J, Baccini $\mathrm{M}$, Vonk $\mathrm{J}$ et al. Acute effects of particulate air 
pollution on respiratory admissions- Results from APHEA 2 project. Am J Respir Crit Care Med $2001 ; 164: 1860-66$.

6. VVAA. El proyecto EMECAM: Estudio español sobre la relación entre la contaminación atmosférica y la mortalidad Rev Esp Salud Pública 1999; $73: 165-314$

7. Ballester F, Sáez M, Perez-Hoyos S, Iñiguez C, Gandarillas A, Tobías A, et al. The EMECAM project: a multicentre study on air pollution and mortality in Spain: combined results for particulates and for sulfur dioxide. Occup Environ Med 2002; 59:300-8.

8. Saez M, Ballester F, Barceló MA, Perez-Hoyos S, Bellido J, Tenias JM et al. A combined analysis of the short-term effects of photochemical air pollutants on mortality within the EMECAM project. Environ Health Perspect 2002; 110:221-0.

9. Ballester F, Iñiguez C, Saez M, Pérez-Hoyos S, Daponte A, Ordóñez JM, et al. Relación a corto plazo de la contaminación atmosférica y la mortalidad en trece ciudades españolas. Med Clin (Barc) 2003; 121:684-689.

10. Ballester F, Saez M, Daponte A, Ordóñez JM, Taracido M, Cambra K et al. Proyecto EMECAS: protocolo del estudio multicéntrico en España de los efectos a corto plazo de la contaminación atmosférica sobre la salud. Rev Esp Salud Pública 2005; 79.

11. WHO. Health Aspects of Air Pollution with Particulate Matter, Ozone and Nitrogen Dioxide. Copenhagen: WHO Regional Office for Europe; 2003 (EUR/03/5042688). Disponible en http://www.euro.who.int/document/e79097.pdf.

12. Diario Oficial de las Comunidades Europeas. Directiva 1999/30/CE relativa a los valores límite de dióxido de azufre, dióxido de nitrógeno, partículas y plomo en el aire ambiente. DOCE núm L163, 29/6/1999.

13. Medina S, Plasència A., Artazcoz L., Quénel P, Katsouyanni K, Mücke HG et al. APHEIS Monitoring the Effects of Air Pollution on Public Health in Europe. Scientific report, 1999-2000. Saint-Maurice: Institut de Vielle Sanitaire; 2001. Disponible en: www.apheis.net

14. WHO. Evaluation and use of epidemiological evidence for Environmental Health Risk Assessment. Copenhagen: WHO Regional Office for Europe; 2000 (EUR/00/5020369). Disponible en: http://www.euro.who.int/document/e68940.pdf
15. WHO. Quantification of health effects of exposure to air pollution. Copenhagen: WHO Regional Office for Europe; 2001 (EUR/01/5026342). Disponible en: http://www.euro.who.int/document/e 74256.pdf.

16. EC Working Group on Particulate Matter. Guidance to member states on PM10 equivalent monitoring and intercomparisons with the reference method. Brussels: Unión Europea; 2002. Didponible en: http://europa.eu.int/comm/environment/air/ pdf/finalwgreporten.pdf.

17. APHEIS 3. Health Impact Assessment of Air Pollution and Comunication Strategy. Third Year Report 2002-2003. Brussels: Unión Europea; 2004. Disponible en http://europa.eu.int/comm/ health/ph_projects/2001/pollution/fp_env_2001_f rep_en.pdf.

18. Atkinson RW, Anderson HR, Medina S, Iñiguez C, Forsberg B et al. Analysis of all-age respiratory hospital admissions and particulate air pollution within the APHEIS programme. En: Apheis 3. Health Impact Assessment of Air Pollution and Comunication Strategy. Third Year Report 20022003. July 2004. Anexes; pp:38-50. Disponible en http://europa.eu.int/comm/health/ph projects/2001/pollution/fp env 2001 frep en.pdf

19. Zanobetti A, Schwartz J, Samoli E. The temporal pattern of mortality responses to air pollution: a multicity assessment of mortality displacement. Epidemiology 2002; 13(1):87-93.

20. Zanobetti A, Schwartz J, Samoli E et al. The temporal pattern of respiratory and heart disease mortality in response to air pollution. Environ Health Perspect 2003; 111(9):1188-93.

21. Schwartz J. Assessing confounding, effect modification, and thresholds in the association between ambient particles and daily deaths. Environ Health Perspect 2000; 108(6): 563-8.

22. Daniels MJ, Dominici F, Samet JM, Zeger SL. Estimating particulate matter-mortality dose-response curves and threshold levels: an analysis of daily time-series for the 20 largest US cities. Am J Epidemiol 2000; 152(5):397-406

23. Institut de Veille Sanitaire. Evaluation de l'impact sanitaire de la pollution atmosphérique urbaine. Version actualisée du guide méthodologique 2003, EIS-PA. Disponible en: http://www.invs.sante.fr/ surveillance/psas9/.

24. Air Quality Health Impact Assessment software AirQ2.2. Disponible en : http://www.euro. 
who.int/eprise/main/WHO/Progs/AIQ/Activities/20040428 2 .

25. Miller BG, Hurley JF. Life table methods for quantitative impact assessments in chronic mortality. J Epidemiol. Community Health 2003; 57:200-6.
26. Medina S, Plasencia A, Ballester F, Mücke HG. Apheis: public health impact of PM10 in 19 European cities. J Epidemiol Community Health 2004; 58:831-6. 